Document downloaded from:

http://hdl.handle.net/10251/65603

This paper must be cited as:

Garcia Chocano, VM.; García Miquel, AH. (2015). DC and AC linear magnetic field sensor based on glass coated amor- phous microwires with Giant Magnetoimpedance. Journal of Magnetism and Magnetic Materials. 378:485-492. doi:10.1016/j.jmmm.2014.11.017.

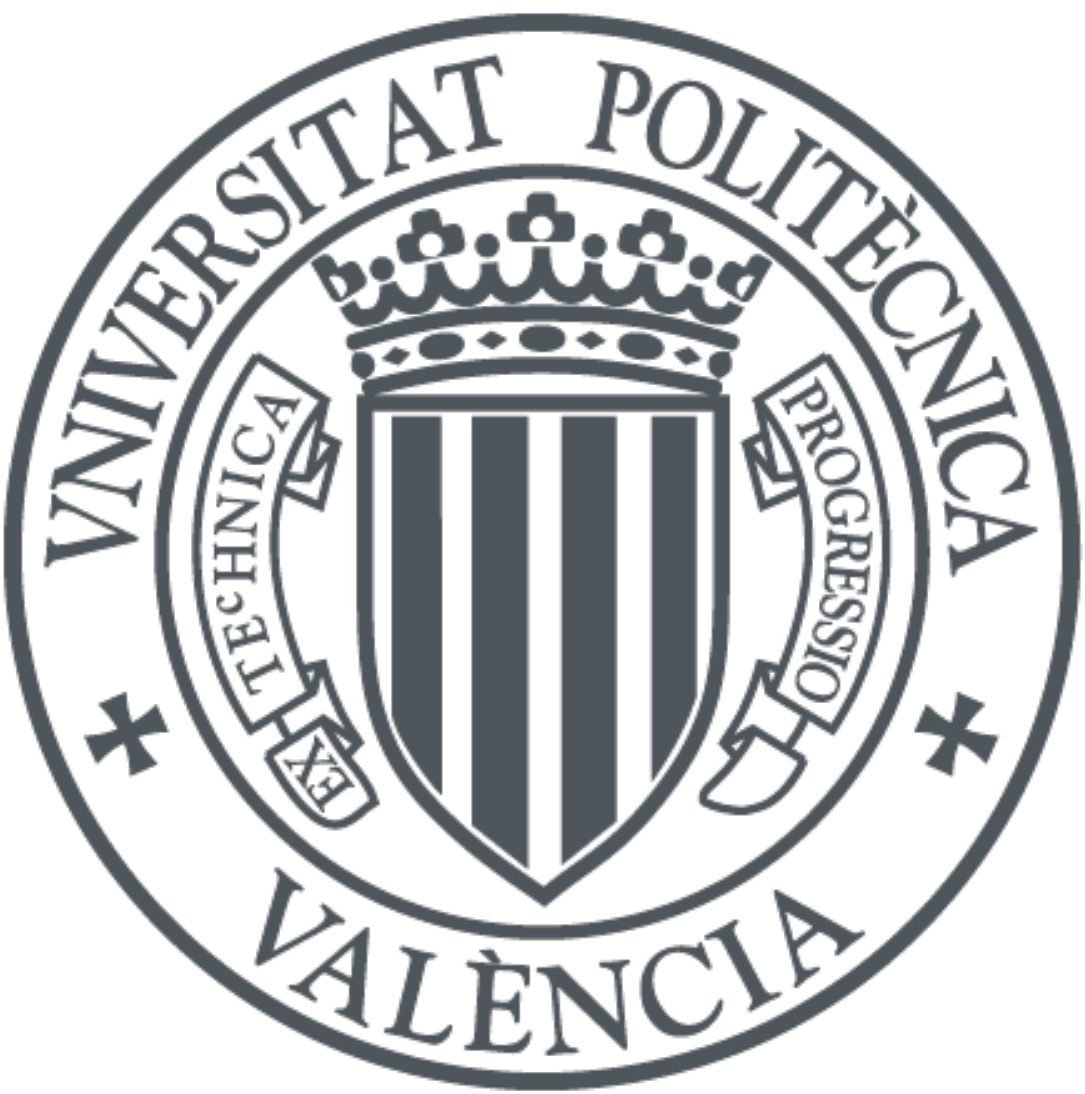

The final publication is available at

http://dx.doi.org/10.1016/j.jmmm.2014.11.017

Copyright Elsevier

Additional Information 


\title{
DC and AC Linear Magnetic Field Sensor Based on Glass Coated Amorphous Microwires with Giant Magnetoimpedance
}

\author{
Víctor Manuel García-Chocano ${ }^{1,3}$ and Héctor García-Miquel ${ }^{2,3}$ \\ ${ }^{1}$ Wave Phenomena Group, Universitat Politécnica de Valencia, 46022 Valencia, Spain. \\ ${ }^{2}$ ITEAM Research Institute, Universitat Politécnica de Valencia, C/Camino de Vera s/n, E-46022 Valencia, Spain. \\ ${ }^{3}$ Electronic Engineering Department, Universitat Politécnica de Valencia, 46022 Valencia, Spain.
}

\author{
hgmiquel@eln.upv.es, vicgarch@teleco.upv.es
}

\begin{abstract}
Giant magnetoimpedance (GMI) effect has been studied in amorphous glass-coated microwires of composition $\left(\mathrm{Fe}_{6} \mathrm{Co}_{94}\right)_{72.5} \mathrm{Si}_{12.5} \mathrm{~B}_{15}$. The impedance of a $1.5 \mathrm{~cm}$ length sample has been characterized by using constant $\mathrm{AC}$ currents in the range of $400 \mu \mathrm{A}$ to $4 \mathrm{~mA}$ at frequencies from 7 to $15 \mathrm{MHz}$ and DC magnetic fields from -900 to $900 \mathrm{~A} / \mathrm{m}$. Double peak responses have been obtained, showing GMI ratios up to $107 \%$. A linear magnetic field sensor for DC and AC field has been designed, using two microwires connected in series with a magnetic bias of $400 \mathrm{~A} / \mathrm{m}$ with opposite direction in each microwire in order to obtain a linear response from \pm 70 $(\mathrm{A} / \mathrm{m})_{\text {rms }}$ for $\mathrm{AC}$ magnetic field, and $\pm 100 \mathrm{~A} / \mathrm{m}$ for DC magnetic field. A closed loop feedback circuit has been implemented to extend the linear range to $\pm 1 \mathrm{kA} / \mathrm{m}$ for DC magnetic field.
\end{abstract}

Keywords: Giant Magnetoimpedance, Amorphous Microwire, Magnetic Field Sensor.

\section{Introduction}

Glass coated amorphous microwires have attracted much attention and they have been intensively studied during the last two decades. Since the Giant Magnetoimpedance (GMI) was discovered in 1994 $[1,2]$, many theoretical models have been developed to explain this phenomenon [3-6]. The magnetization processes in microwires showing the GMI effect have been studied $[7,8]$ and many works dealing with technological applications were reported because of the outstanding magnetic properties [9-15] which makes them useful as high sensitivity magnetic sensors [1620], biosensors [17,19,21,22], current sensors [23] and magnetometers [24]. It is remarkable the contribution of Mohri et al. in the development of electronic circuits for GMI sensors [16,20]. These previous works reported GMI sensors including signal conditioning. Such sensors were excited with a pulsed voltage, leading to changes in the current amplitude which modifies de characterized GMI response which is done at constant current amplitude (note that the GMI ratio and the peak of the GMI response depend on the current). Here we present a different approach where the GMI sensor is excited with constant current amplitude. We also apply a bias field in order to work on the field over the GMI peak, where the hysteresis is negligible. GMI in amorphous microwires has been also studied at microwave frequencies [15,25], and also the absorptive properties due to the phenomenon of Ferromagnetic Resonance (FMR) [26-29], or the recently experimental demonstration of its behavior as a metamaterial due to the coexistence of negative permittivity and permeability in the frequency region between FMR and Ferromagnetic Antiresonance (FMAR) [30]. This structure allows the possibility of modifying a pass band between FMR and FMAR through an external magnetic field [30] or with a current though the microwire [31], even modulating a microwave signal with an AC current.

GMI has been studied in several structures like magnetic ribbons, wires and microwires [32-33]. The GMI phenomenon is due to the skin effect in magnetic conductors. When an external magnetic field interacts with the conductor, the permeability is highly increased in such a manner that the skin depth is reduced. The impedance of a magnetic conductor with radius $r$ and an AC driving current is given by [4]:

$$
Z=R_{d c}(k r) \frac{J_{o}(k r)}{2 J_{1}(k r)},
$$

where $R_{d c}$ is the DC resistance and $J_{o}$ and $J_{1}$ are the Bessel functions of zeroth and first order, respectively. 
In addition, $k=(1+j) / \delta$, where $\delta$ is the skin depth given by

$$
\delta=\frac{1}{\sqrt{\pi f \sigma \mu_{\phi}}},
$$

$\sigma$ being the electrical conductivity, $\mu_{\emptyset}$ the magnetic circular permeability and $f$ the frequency of the AC current.

The high value of $\mu$ in soft magnetic materials will cause that the skin effect appears at lower frequencies than nonmagnetic metals with the same conductivity. In addition, its strong dependence with the external magnetic field will originate large impedance variations. In ferromagnetic materials the $\mathrm{AC}$ permeability is not isotropic and depends on the orientation of the $\mathrm{AC}$ and $\mathrm{DC}$ magnetic fields and the sample anisotropies. Concretely it is the effective transverse permeability $\mu_{\mathrm{t}}$ which is involved in GMI, also called circular permeability $\mu_{\phi}$ in the case of cylindrical geometry.

The impedance variations are usually quantified through the GMI ratio, which is defined as:

$$
\frac{\Delta Z}{Z}(H)=\frac{|Z(H)|-\left|Z\left(H_{\max }\right)\right|}{\left|Z\left(H_{\max }\right)\right|}
$$

GMI ratios can typically exceed the value of $100 \%$, some works reporting values up to $600 \%$ [21]. Due to these huge impedance variations, which occur in a narrow range of magnetic fields, microwires can be employed to design highly sensitive magnetic sensors. However the main drawback associated to this phenomenon consists of the non-linear behavior of the samples.

This work is focused on the GMI phenomenon in glass coated amorphous microwires whose diameters take typical values around $10 \mu \mathrm{m}$. Firstly these microwires have been characterized in a system designed to measure the impedance of magnetic samples under different magnetic fields and excitation currents. Then, we present the design and test of a DC and $\mathrm{AC}$ linear magnetic field sensor intended to measure fields in the range $\mathrm{H}_{\mathrm{dc}}= \pm 100 \mathrm{~A} / \mathrm{m}$ and $\mathrm{H}_{\mathrm{ac}, \mathrm{RMS}}$ $=70 \mathrm{~A} / \mathrm{m}$ with a frequency range up to $50 \mathrm{kHz}$. Finally, we propose an upgrade of the initial sensor which allows to extent the measurable range of DC fields up to $1 \mathrm{kA} / \mathrm{m}$ with a remarkable linearity.

\section{GMI measurement procedure}

As exposed, the GMI phenomenon is a consequence of the skin depth, which depends on the frequency and the circular permeability of the magnetic sample. This last parameter in turn depends on the amplitude and frequency of the electric current flowing through the sample, as well as the presence of an external magnetic field. Therefore the experimental characterization of the GMI phenomenon relies in the implementation of an automated system capable of controlling these three parameters. In this sense a measurement setup intended to measure the impedance of the samples under different conditions has been developed.

Fig. 1 shows the equipment included in the system. Three perpendicular Helmholtz coils are used in order to compensate the Earth's magnetic field while an additional coil is employed to generate the magnetic field which excites the samples. The DC current feeding this latter coil is provided through a KEPCO BOP 50-8D current source and the amplitude of the magnetic field applied to the samples is measured with a FW Bell 7010 Gaussmeter. On the other hand, the current passing through the microwire consists of a sinusoidal wave with fixed current amplitude which is generated with an Agilent 33120 function generator. Finally, the electrical parameters are acquired through a Tektronix TDS3012B digital oscilloscope. All these devices are connected with a GPIB bus to a computer.

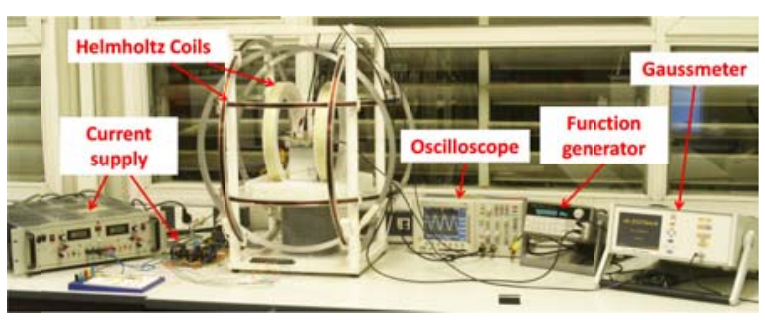

Figure 1: GMI measurement setup containing the equipment required to characterize magnetic samples.

The impedance of the microwires is obtained through the scheme represented in Fig. 2. It is based on a non-inductive precision resistor $\mathrm{R}_{\mathrm{ref}}$ in series with the sample. Thus the current through the sample can be determined by measuring the voltage drop across such resistor with a differential probe. Then the voltage drop across the sample is directly obtained, in such a manner that the unknown impedance is calculated as $\mathrm{Z}=\mathrm{V}_{1} \cdot \mathrm{R}_{\mathrm{ref}} / \mathrm{V}_{2}$. Note that this process is performed by setting a current with constant amplitude. Since the function generator cannot provide constant current amplitude, the computer controlling the system sets the voltage $\mathrm{V}_{\text {in }}$ according to the expected value of $\mathrm{V}_{1}$ for the desired measurement current amplitude. 


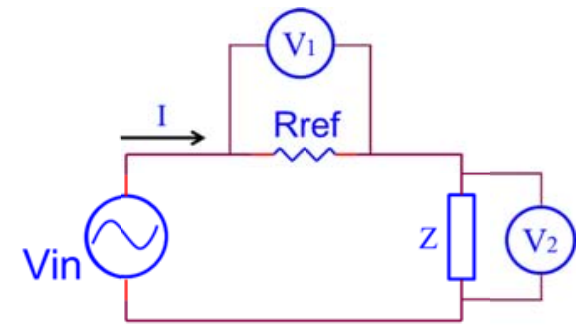

Figure 2: Circuit employed to determine the impedance of the samples.

\section{Experimental results}

The amorphous microwires considered in this work have $\left(\mathrm{Fe}_{6} \mathrm{Co}_{94}\right)_{72.5} \mathrm{Si}_{12.5} \mathrm{~B}_{15}$ composition, which is characterized by near zero magnetostriction $\left(\lambda_{s}=\right.$ $-10^{-7}$ ) [10]. The microwires used for the sensor are $1.5 \mathrm{~cm}$ long, and have a ferromagnetic core with $4 \mu \mathrm{m}$ of diameter surrounded with a glass coating of $5 \mu \mathrm{m}$ (see Fig.3). The Co-rich composition tied with its null magnetostriction constant creates a circular magnetic domain structure that shows GMI, characterized by a double peak response [18].

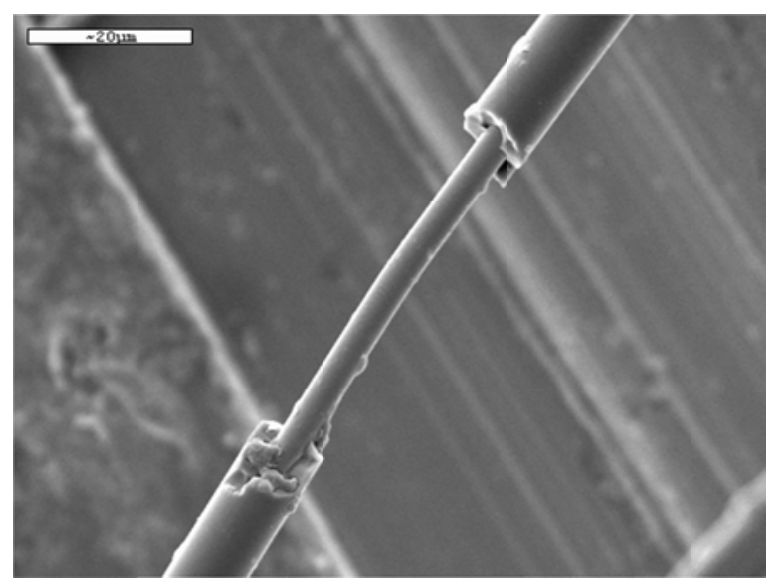

Figure 3: SEM image of a magnetic microwire. It is based on a magnetic core which is covered with a glass coating.

The measures were performed for a microwire with a length of $1.5 \mathrm{~cm}$ under static magnetic fields in the range $\pm 900 \mathrm{~A} / \mathrm{m}$, frequencies from 7 to $15 \mathrm{MHz}$ and currents up to $4 \mathrm{~mA}_{\mathrm{p}}$. Fig. 4 shows the impedance modulus of the increasing branch of the GMI curve for different frequencies and a constant current of $1 \mathrm{~mA}_{\mathrm{p}}$. As expected, it is observed a double peak behavior due to the circular anisotropy field, $\mathrm{H}_{\mathrm{K}}$, characterizing the employed microwires. Such peaks appear when the applied axial field equals the anisotropy field $\pm \mathrm{H}_{\mathrm{K}}$, giving a maximum in the circular permeability that leads to a minimum in the skin depth and therefore a maximum in impedance. As can be seen in Fig.4, the circular anisotropy field $\mathrm{H}_{\mathrm{K}}$, shifts towards higher magnetic field when frequency is increased. It is worth to note the strong dependence of the impedance around $\pm \mathrm{H}_{\mathrm{K}}$ for extremely low variations of the external magnetic field. The values of impedance are similar for high magnetic fields (saturation zone), although the amplitude of the peaks changes with the frequency. Thus the GMI ratio will be greater as the frequency increases for the frequency range here considered.

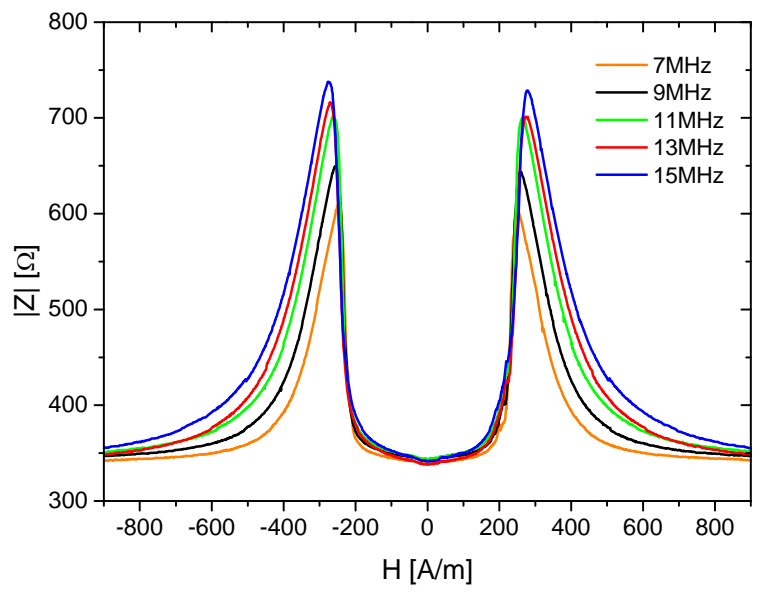

Figure 4: Impedance modulus of the sample under study as a function of the magnetic field with a constant current of $1 \mathrm{~mA}_{\mathrm{p}}$. Each curve corresponds to a particular frequency (see the legend) in the frequency range of 7 to $15 \mathrm{MHz}$.

Fig. 5 shows a current sweep from $0.4 \mathrm{~mA}_{\mathrm{p}}$ to $4 \mathrm{~mA}_{\mathrm{p}}$ performed at 7 and $15 \mathrm{MHz}$. It is observed the double peak response although, unlike the last figure, a remarkable dependence between the current and the position of the peaks is found. As shown, the positon of the peak moves to lower magnetic field as the current is increased. This trend can be observed in Fig. 6 , which shows the position of the peaks for different frequencies as a function of the current. It is found that the position of the peaks is approximately the same at low currents disregarding the frequency, although at higher currents the differences are more pronounced. As previously mentioned, the higher the frequency, the higher the magnetic field where the peak occurs.

The GMI ratio of the whole set of data has been calculated and represented in Fig. 7. To ensure that the impedance corresponding to the saturated field is reached, these values were measured at $1200 \mathrm{~A} / \mathrm{m}$. It is observed a behavior showing a maximum around $1 \mathrm{~mA}_{\mathrm{p}}$ for all the frequencies. As shown, the GMI ratio increases with the frequency, reaching a maximum value of $107 \%$ at $15 \mathrm{MHz}$ for $1 \mathrm{~mA}_{\mathrm{p}}$. In order to 
examine the maximum GMI in more detail, a cubic spline interpolation has been included for the curve of GMI ratio at $15 \mathrm{MHz}$. It is found that the maximum occurs at $1.16 \mathrm{~mA}_{\mathrm{p}}$ where a GMI ratio of $109 \%$ is achieved.

An important feature of any sensor consists of having a low hysteresis behavior in its measurement range. Therefore, the hysteresis of the sample has been characterized through a measure where the field is increased from $-1000 \mathrm{~A} / \mathrm{m}$ to $1000 \mathrm{~A} / \mathrm{m}$ (increasing branch) and then it is decreased to $-1000 \mathrm{~A} / \mathrm{m}$ (decreasing branch). The impedance obtained along these two branches is represented in Fig. 8(a). It is worth to note that the hysteresis has a negligible effect for magnetic fields higher than the position of the GMI peaks, which corresponds to the range where the microwires of the developed sensor will work. To emphasize this fact, a zoomed view is presented in Fig. 8(b). Here, the increasing and decreasing branches differ in less than $3 \Omega$ at fields beyond $-300 \mathrm{~A} / \mathrm{m}$. Note that these curves correspond to a test between $\pm 1000 \mathrm{~A} / \mathrm{m}$ and in practice the dynamic range of the sensor will be lower. In any case, it is possible to generate a reset magnetic pulse to avoid the hysteresis (it could be performed with the bias coils that will be introduced later).

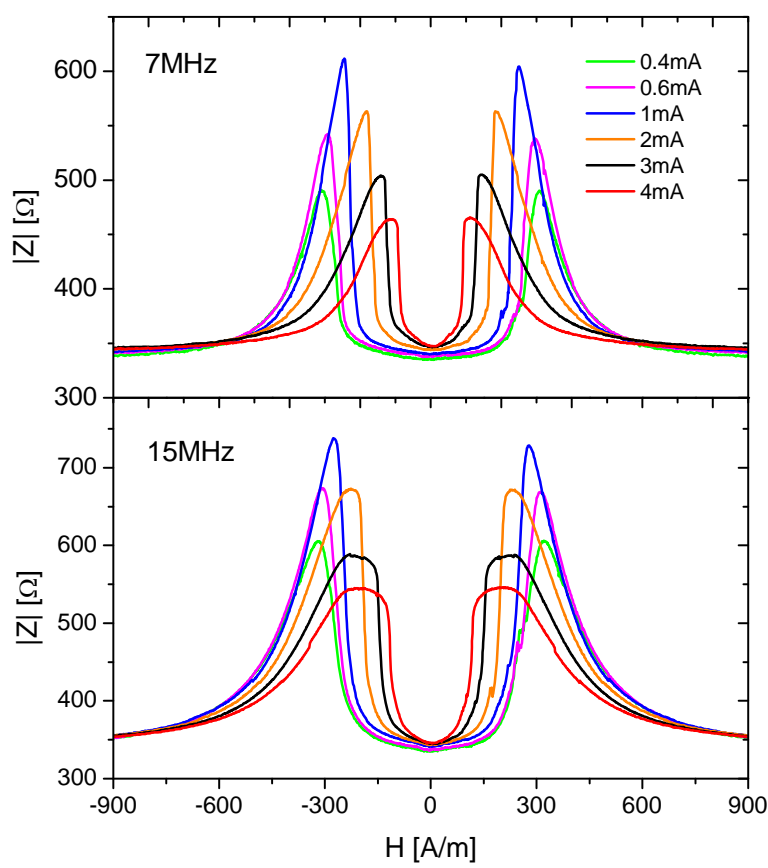

Figure 5: Impedance modulus of the sample at 7 and $15 \mathrm{MHz}$ for currents from $0.4 \mathrm{~mA}_{\mathrm{p}}$ to $4 \mathrm{~mA}_{\mathrm{p}}$.

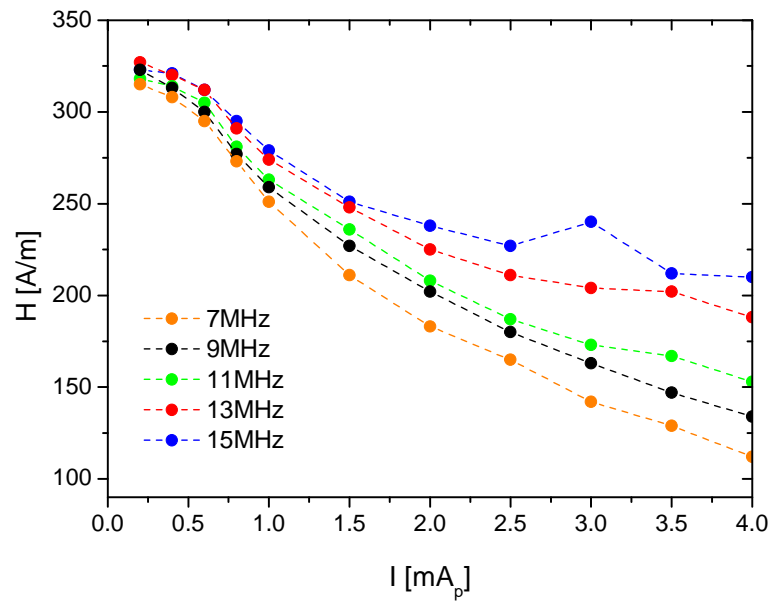

Figure 6: Magnetic field where maximum impedance is found as a function of the $\mathrm{AC}$ current through the microwire and the frequency.

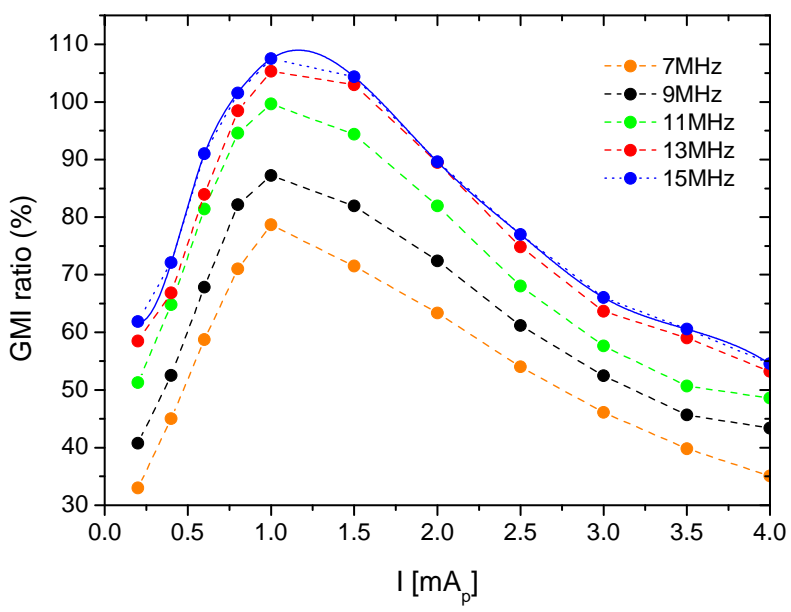

Figure 7: GMI ratio as a function of the AC current exciting the microwire at several frequencies. The continuous line corresponds to a cubic spline interpolation of the GMI ratio at $15 \mathrm{MHz}$. The impedance of the sample of the saturated zone was obtained with a field of $1200 \mathrm{~A} / \mathrm{m}$. 

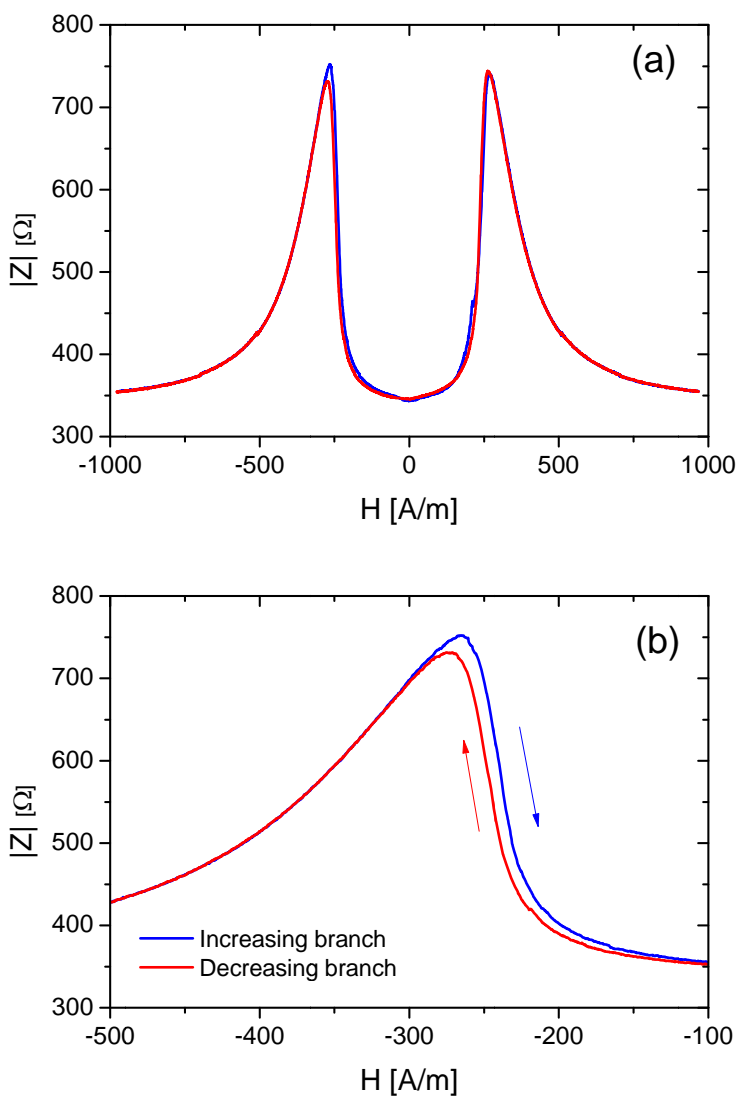

Figure 8: (a) Characterization of the hysteresis of the microwire through a measurement of the increasing and decreasing branches in the range $\pm 1000 \mathrm{~A} / \mathrm{m}$ at $15 \mathrm{MHz}$ and $1 \mathrm{mAp}$, (b) zoom from $-500 \mathrm{~A} / \mathrm{m}$ to $-100 \mathrm{~A} / \mathrm{m}$. The arrows indicate the sense of the magnetic field sweep employed in the test.

\section{Magnetic sensor design}

As shown, the GMI phenomenon provides large variations of impedance for low magnetic fields, leading to the implementation of sensors with high sensitivity. However this effect is also highly nonlinear and some circuitry is required in order to get a linear response. For this purpose, consider the range of magnetic fields between the impedance maximum and the saturation regime. In this region there is a zone which is almost linear. This can be even used in applications where the linearity is not a critical parameter. However, the linearity can be improved by combining the response of two microwires [16]. This process requires that the responses of the microwires are shifted by means of two bias fields with opposite directions. This can be easily obtained by using two coils configured in series-opposition, as shown in Fig. 9. Finally, the linearized response is obtained through the difference of both responses. Fig. 10 shows schematically this procedure. Here the red and green curves represent the impedances of the microwires shifted by a bias field of $\pm 400 \mathrm{~A} / \mathrm{m}$. The blue curve corresponds to the difference of voltage of the two microwires, where a highly linear response can be observed.

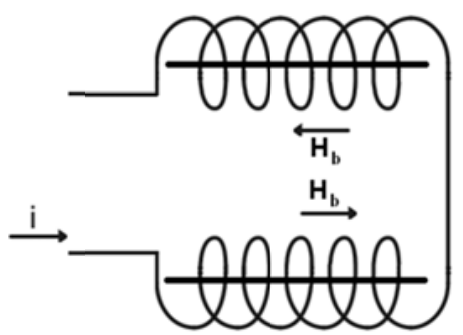

Figure 9: DC bias field coils connected in series-opposition, intended to displace the GMI response of two microwires in opposite directions.

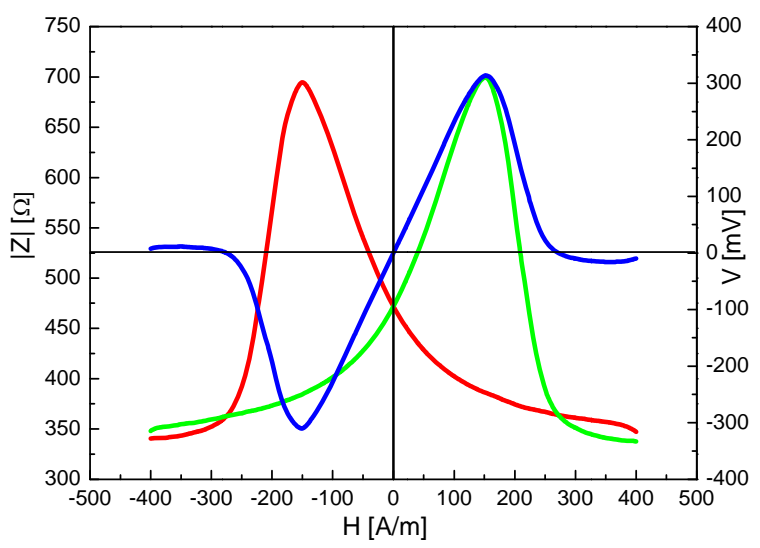

Figure 10: Linearized response (blue curve) of impedance obtained by subtracting the GMI response of two microwires with magnetic polarization of $-400 \mathrm{~A} / \mathrm{m}$ (red curve) and $+400 \mathrm{~A} / \mathrm{m}$ (green curve). The right scale represents the corresponding voltage when considering a current of $1 \mathrm{~mA}_{\mathrm{p}}$ through the sample.

In general terms, the required circuitry is based on the block diagram of Fig. 11. It begins with an AC current source which excites both microwires, each one polarized with a DC bias magnetic field. Since we are interested in the amplitude of the impedance, the voltage drop of the microwires is led to an envelope detector. Finally, the two responses are subtracted with a differential amplifier which also sets the desired gain to tune the overall sensibility of the sensor.

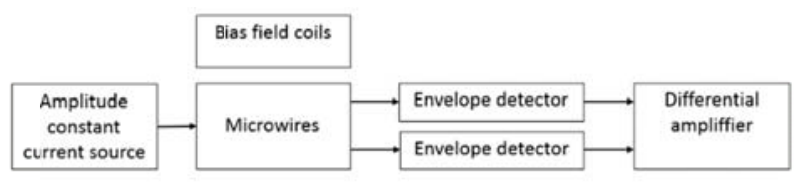


Figure 11: General block diagram of a sensor circuit linearizing the response amorphous microwires.

An implementation of the circuit based on the diagram of Fig. 11 is shown in Fig. 12. Here the current sources are implemented with high frequency operational amplifiers (LM7171), in such a manner that the voltage drop in the microwires corresponds to the output of such operational amplifiers. The envelope detector is made with a diode followed by a resistor in parallel with a capacitor. In order to compensate the voltage drop of the diode, it has been included a bias network based on a voltage divider. The output signals of the envelope detectors drive the inputs of a differential amplifier. The output of such amplifier is low-pass filtered for the analysis of DC fields. It can be also led to a circuit which obtains either its amplitude or RMS value when considering AC fields. The bottom circuit of Fig. 12 represents the DC current source feeding the two coils which provide the bias fields of the microwires.

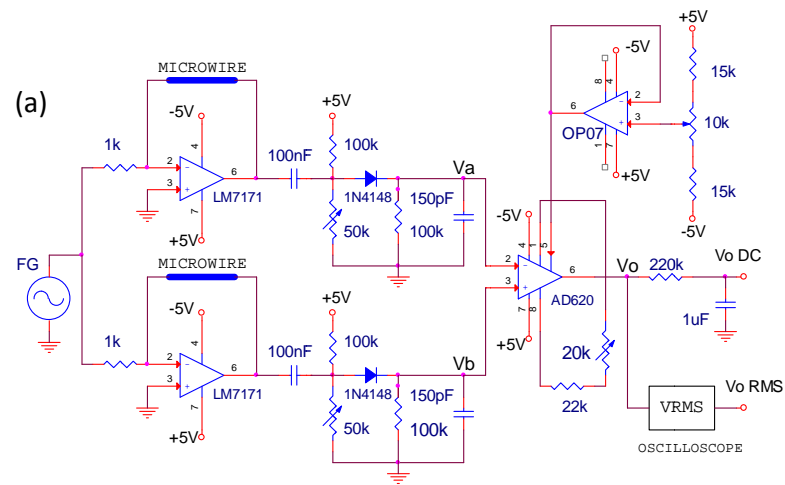

(b)

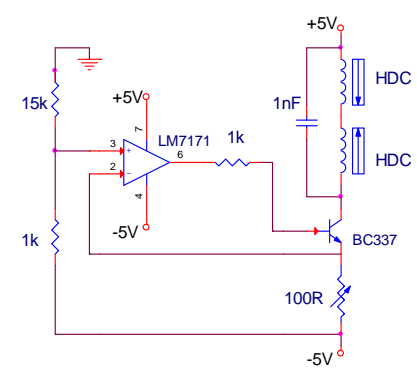

Figure 12: (a) Signal conditioning circuit for microwirebased sensor and (b) circuit for polarization of the microwires with a constant magnetic field of $\pm 400 \mathrm{~A} / \mathrm{m}$.

\section{Sensor characterization}

The circuit from Fig. 12 has been built and tested in the experimental setup of Fig. 2. Since it has been tested under DC but also AC magnetic fields, the Helmholtz coils were connected to an Agilent 33120 function generator followed by a current buffer.

In order to show experimentally the linearization process explained in the above section, a magnetic field with triangular waveform and frequency $300 \mathrm{~Hz}$ has been applied to the sample while the voltages $\mathrm{V}_{\mathrm{a}}$, $\mathrm{V}_{\mathrm{b}}$ and $\mathrm{V}_{\mathrm{o}}$ (see Fig. 11) are recorded. Fig. 13 shows a screenshot of the oscilloscope measuring the voltage $\mathrm{V}_{\mathrm{a}}$ when the bias coils of the microwires are disconnected. Note that the signal follows the shape of the impedance of the microwire. This behavior is due to the fact that the signal $\mathrm{V}_{\mathrm{a}}$ is the envelope of the voltage drop across the microwire. When the bias field is applied to the microwires, the voltages $\mathrm{V}_{\mathrm{a}}, \mathrm{V}_{\mathrm{b}}$ and $\mathrm{V}_{\mathrm{o}}$ take the form of the yellow, blue and red curves from Fig. 14, respectively. This figure can be compared with Fig. 10 and shows the good operation of the device and the linearization process. Note that in these cases the amplitude of the magnetic field exceeds the linear range of the sensor in order to show the impedance curves for a broad range of fields. When the amplitude is limited to the linear range of the sensor, the magnetic field is accurately acquired. Its shape, corresponding to a triangular waveform is shown in Fig. 15.

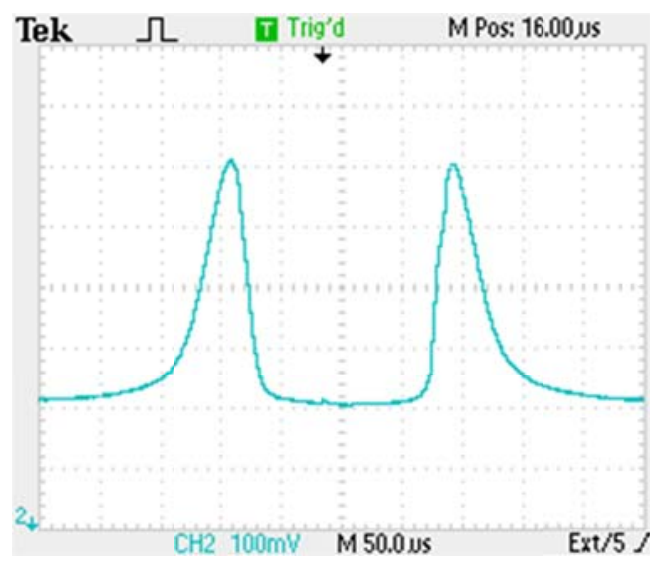

Figure 13: screenshot of the oscilloscope measuring the voltage $\mathrm{V}_{\mathrm{a}}$ in the envelope detector when the bias coils of the microwires are disconnected and a magnetic field with triangular waveform is applied. 


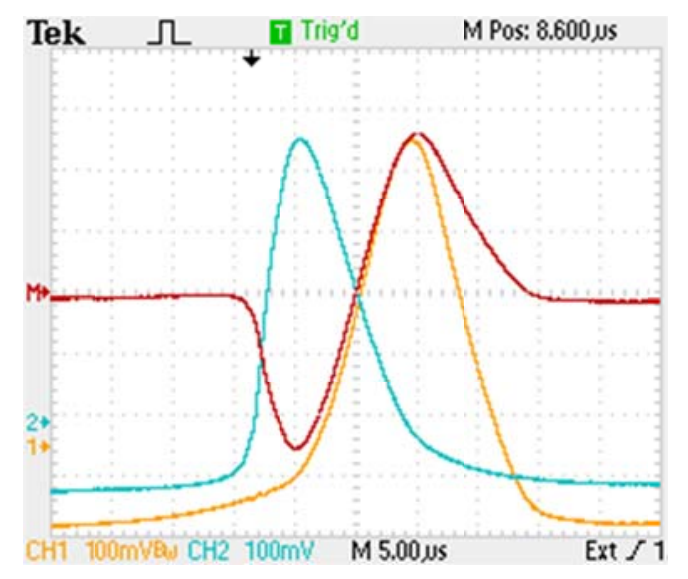

Figure 14: GMI screenshot of the oscilloscope measuring the voltages $V_{a}$ and $V_{b}$ after the envelope detector (blue and yellow curves), and the voltage difference between them obtained through the mathematical function of the oscilloscope (red curve).

The DC and AC responses of the sensor have been characterized through a sweep of external magnetic field. The parameter here analyzed corresponds to the linearity, which is obtained by comparing the acquired data with a linear regression. The measured DC response is shown in Fig. 16 with red dots. The blue line corresponds to the linear regression of such data. The maximum linearity error between the response of the sensor and the linear regression is $3 \%$ for a measurable range of $\pm 100 \mathrm{~A} / \mathrm{m}$, with a sensitivity of $10 \mathrm{mV} /(\mathrm{A} / \mathrm{m})$.

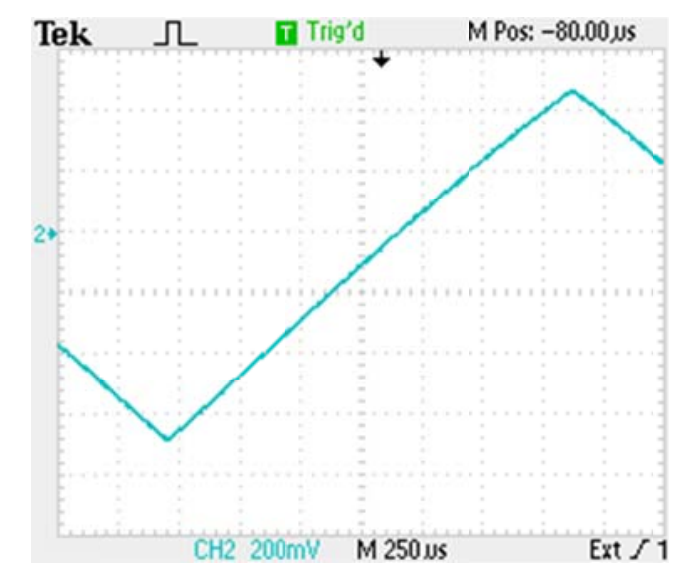

Figure 15: measured curve at the output $\left(\mathrm{V}_{\mathrm{o}}\right)$ for a triangular magnetic field with a frequency of $300 \mathrm{~Hz}$ and an amplitude of $70 \mathrm{~A} / \mathrm{m}(\mathrm{rms})$.

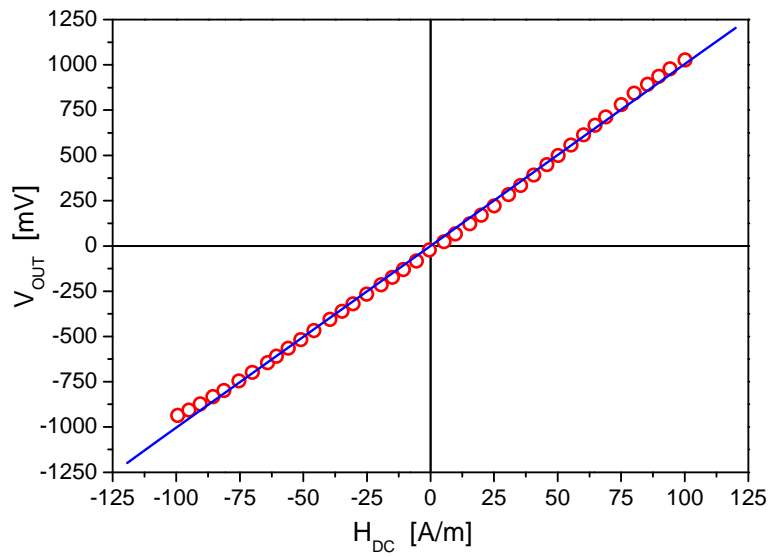

Figure 16: Measured DC response of the sensor for a measurable range of $\pm 100 \mathrm{~A} / \mathrm{m}$, with a sensitivity of $10 \mathrm{mV} /(\mathrm{A} / \mathrm{m})$ and a maximum linearity error of $3 \%$.

With regard to the AC response, Fig. 17 shows the measured AC response for a measurable range of $70 \mathrm{~A} / \mathrm{m}$ (rms), for frequencies from $1 \mathrm{kHz}$ to $100 \mathrm{kHz}$ with a sensitivity of $10 \mathrm{mV} /(\mathrm{A} / \mathrm{m})$. The linearity error found in these data corresponds to $3 \%$ for frequencies lower than $50 \mathrm{kHz}$. It is observed that the response of the sensor saturates for higher frequencies, which reduces the measurable range of magnetic fields.

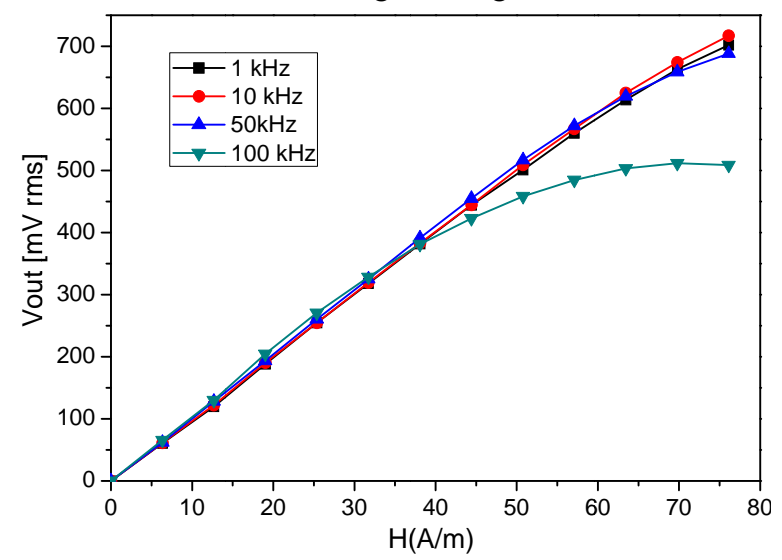

Figure 17: Measured $\mathrm{AC}$ response for a measurable range of $70 \mathrm{~A} / \mathrm{m}(\mathrm{rms})$, with a sensitivity of $10 \mathrm{mV} /(\mathrm{A} / \mathrm{m})$ and a maximum linearity error of $3 \%$ (up to $50 \mathrm{kHz}$ ).

As shown, we have designed a GMI sensor presenting interesting features over commercially available magnetic field sensors due to its higher sensitivity and negligible hysteresis. Table 1 shows a comparison between the developed sensor and other sensing technologies. Anisotropic Magneto Resistance (AMR) sensors provide good hysteresis, but their sensitivity is two orders of magnitude below GMI sensors. On the other hand, Giant Magneto Resistance (GMR) sensors have a sensitivity one order of 
magnitude below GMI sensors, and they have a significant hysteresis effect and a worse response for magnetic fields below $50 \mathrm{~A} / \mathrm{m}$. As a drawback, the GMI sensor has proved to work within a lower frequency range, although higher bandwidths can be achieved by improving the signal conditioning circuit.

\begin{tabular}{|c|c|l|c|}
\hline $\begin{array}{l}\text { Sensor } \\
\text { technology }\end{array}$ & $\begin{array}{c}\text { Sensitivity } \\
\mathbf{m V} /(\mathbf{A} / \mathbf{m})\end{array}$ & Properties & $\begin{array}{l}\text { Frequency } \\
\text { range }\end{array}$ \\
\hline $\begin{array}{c}\text { AMR } \\
\text { bridge }\end{array}$ & 0.1 & $\begin{array}{l}\text { Good linearity, } \\
\text { low hysteresis }\end{array}$ & $1 \mathrm{MHz}$ \\
\hline $\begin{array}{c}\text { GMR } \\
\text { bridge }\end{array}$ & 1 & $\begin{array}{l}\text { High hysteresis, } \\
\mathrm{H}_{\text {min }} \approx 50 \mathrm{~A} / \mathrm{m}\end{array}$ & $1 \mathrm{MHz}$ \\
\hline GMI & 10 & $\begin{array}{l}\text { Negligible } \\
\text { hysteresis, good } \\
\text { linearity and } \\
\text { accuracy in the } \\
\text { range } 0-100 \mathrm{~A} / \mathrm{m}\end{array}$ & $50 \mathrm{KHz}$ \\
\hline
\end{tabular}

Table 1: Comparison of the main features of the designed sensor and other sensing technologies.

\section{High field measurement}

The circuit shown in Fig. 12 can be easily improved to extend the range of measurable fields. For this purpose it has been added a feedback coil which covers both microwires with their respective bias coils. This new compensation coil generates a magnetic field which counteracts the external field in such a manner that the net magnetic field is zero, and the microwires remains biased at their bias field of $\pm 400 \mathrm{~A} / \mathrm{m}$.

The differential amplifier has been replaced by a current feedback amplifier (LT1210), the output of the envelope detectors feeding the inputs of this amplifier. The output of the amplifier is connected to a resistor in series with the compensation coil, thus providing a negative feedback to the circuit. The voltage drop in this resistor is proportional to the applied magnetic field by the feedback coil in order that the inputs of the operational amplifier are virtually short cut, which means that both microwires work at the point of null field in Fig. 10. In principle, the only limitation of this implementation concerning the maximum measurable field is the output current capability of the amplifier. Note that the additional coil could be obviated if the feedback is directly introduced through the bias coils, although in this case some circuitry would be required to mix the currents corresponding to the feedback and the bias fields.

The schematic of this proposal is represented in Fig.18. This circuit has been build and tested for DC magnetic fields. AC fields have been not considered since the oscillating signal through the compensation coil induces a voltage on the bias coils, since it acts as a transformer. Fig.19 shows the DC response of the sensor. It is found a linear operation in the range $\pm 1 \mathrm{kA} / \mathrm{m}$, much higher than that reported in the previous section. In addition the linearity error is within $0.8 \%$. At higher magnetic fields, the operational amplifier cannot provide enough current to feed the compensation coil. This fact suppresses the negative feedback of the circuit and the sensor no longer measure properly.

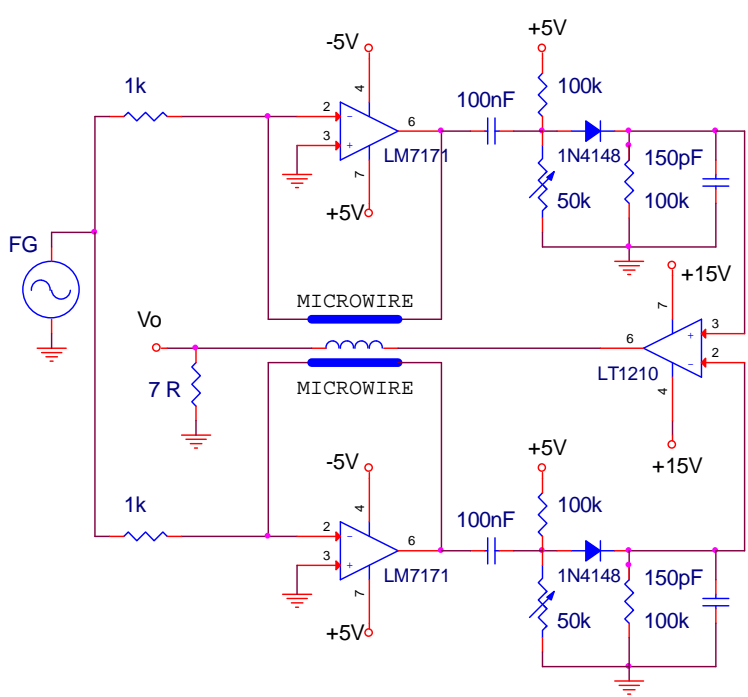

Figure 18: Circuit with a feedback coil in order to extent the measurement range up to $\pm 1 \mathrm{kA} / \mathrm{m}$.

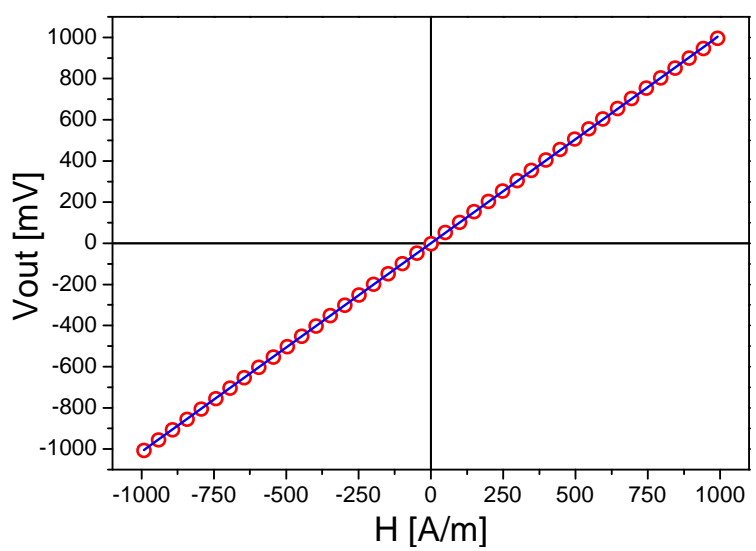

Figure 19: DC response of the sensor, with the feedback coil in the range $\pm 1 \mathrm{kA} / \mathrm{m}$. The linearity error is within $0.8 \%$.

\section{Conclusions}


GMI effect has been studied in glass-coated amorphous microwires with composition $\left(\mathrm{Fe}_{6} \mathrm{Co}_{94}\right)_{72.5} \mathrm{Si}_{12.5} \mathrm{~B}_{15}$. An experimental setup intended to measure the impedance of the microwires under different AC currents and magnetic fields has been developed and employed to characterize the samples in the $7-15 \mathrm{MHz}$ frequency range and currents from 0.4 to $4 \mathrm{~mA}_{\mathrm{p}}$. The GMI ratio has been obtained from the acquired data, showing that it reaches a maximum for a specific current. The maximum GMI ratio obtained during the tests was $107 \%$ for $1 \mathrm{~mA}_{P}$ and $15 \mathrm{MHz}$.

By using the information of the experimental characterization of the microwires, we have designed a magnetic field sensor. Despite the highly non-linear behavior of the microwire impedance, it is shown that a linear response can be achieved through the combination of two microwires with two opposite bias fields. In this manner, a highly sensitive magnetic field sensor has been built and tested. Experimental measures show a good linearity for $\mathrm{DC}$ and $\mathrm{AC}$ with amplitudes of $100 \mathrm{~A} / \mathrm{m}$ and frequencies up to $50 \mathrm{kHz}$. The achieved sensitivity of $10 \mathrm{mV} /(\mathrm{A} / \mathrm{m})$ is much higher than those of conventional magnetic field sensors. In addition, we have extended the linear range for DC fields up to $1 \mathrm{kA} / \mathrm{m}$ by introducing a feedback coil which keeps the microwires at fields below the limit of their linear response.

\section{References}

[1] R. S. Beach and A. E. Berkowitz. Giant magnetic field dependent impedance of amorphous $\mathrm{FeCoSiB}$ wire. Appl. Phys. Lett. 64 (1994) 3652-3654.

[2] K.V. Rao, F.B. Humphrey, J.L.Costakramer. Very large magneto-impedance in amorphous soft ferromagnetic wires. J. Appl. Phys. 76 (1994) 62046208 .

[3] L. Kraus. Theory of giant magneto-impedance in the planar conductor with uniaxial magnetic anisotropy. J. Magn. Magn. Mater. 195 (1999) 764778.

[4] Chen D.-X., Munoz J.L., Hernando A. Vazquez M. Magnetoimpedance of metallic ferromagnetic wires. Phys. Rev. B 57 (1998) 10699-10704.

[5] B. Dufay, S. Saez, C. Dolabdjian, A. Yelon, D. Menard. Physical properties and giant magnetoimpedance sensitivity of rapidly solidified magnetic microwires. J. Magn. Magn. Mater. 324 (2012) 2091-2099.

[6] N.A Usov, A.S. Antonov, A.N. Lagar'kov. Theory of giant magneto-impedance effect in amorphous wires with different types of magnetic anisotropy. J. Magn. Magn. Mater. 185 (1998) 159-173.

[7]J.M. Blanco, A. Zhukov, J. Gonzalez. Asymmetric torsion stress giant magnetoimpedance in nearly zero magnetostrictive amorphous wires. J. Appl. Phys. 87 (2000) 4813-4815.

[8] M. Ipatov, V. Zhukova, J. Gonzalez, A. Zhukov, Manipulating the magnetoimpedance by $\mathrm{dc}$ bias current in amorphous microwire. J. Magn. Magn. Mater. 324 (2012) 4078-4083.

[9] M. Knobel, K.R. Pirota. Giant magnetoimpedance: concepts and recent progress. J. Magn. Magn. Mater. 242-245 (2002) 33-40.

[10] L. V. Panina and K. Mohri. Magneto-impedance effect in amorphous wires. Appl. Phys. Lett. 65 (1994) $1189-1191$.

[11] H. García-Miquel, D. X. Chen, M. Vázquez. Domain wall propagation in bistable amorphous wires. J. Magn. Magn. Mater. 212 (2000) 101-106.

[12]G.V. Kurlyandskaya, N.G. Bebenin. V.O. Vas'kovskii. Giant magnetic impedance of wires with a thin magnetic coating. Phys. Met. Metallogr. 111 (2011) 133-154.

[13] R. Varga, P. Vojtaník, H. A. Davies. Low-field magnetoimpedance of amorphous $\mathrm{CoFeSiB}$ alloy wire. J. Magn. Magn. Mater. 261 (2003) 360-368.

[14] K. R. Pirota, L. Kraus, H. Chiriac, M. Knobel. Magnetic properties and giant magnetoimpedance in a $\mathrm{CoFeSiB}$ glass-covered microwire. J. Magn. Magn. Mater. 221 (2000) 243-247.

[15] J. M. García-Beneytez, F. Vinai, L. Brunetti, H. García Miquel, M. Vázquez. Study of magneto impedance effect in the microwave frequency range for soft magnetic wires and microwires. Sensors \& Actuators A 81 (2000) 78-81

[16] K. Mohri, L.V. Panina, T. Uchiyama, K. Bushida, $M$. Noda. Sensitive and quick response micro magnetic sensor utilizing magneto-impedance in Co-rich amorphous wires. IEEE Trans. Magn. 31 (1995) 12661275.

[17] G. V. Kurlyandskaya. Giant Magnetoimpedance for biosensing: Advantages and shortcomings. J. Magn. Magn. Mater. 321 (2009) 659-662.

[18] G.V. Kurlyandskaya, A. García-Arrobas, J.M. Barandiaran. Advantages of nonlinear giant magnetoimpedance for sensor applications. Sensors \& Actuators A 106 (2003) 234-239.

[19] P. Marin, M. Marcos, A. Hernando. High magnetomechanical coupling on magnetic microwire for sensors with biological applications. Appl. Phys. Lett. 96 (2010) 262512.

[20] K. Mohri, T. Uchiyama, L.P. Shen, C.M. Cai, L.V. Panina. Sensitive micro magnetic sensor family utilizing magneto-impedance (MI) and stressimpedance (SI) effects for intelligent measurements and controls. Sensors \& Actuators A 91 (2001) 85-90.

[21] H. Chiriac, M. Tibu, A.E. Moga, D.D. Herea. Magnetic GMI sensor for detection of biomolecules. J. Magn. Magn. Mater. 293 (2005) 671-676. 
[22] J. Llandro, J.J. Palfreyman, A. Ionescu, C.H.W. Barnes. Magnetic biosensor technologies for medical applications: a review. Med. Biol. Eng. Comput. 48 (2010) 977-998.

[23] Bing Han, Tao Zhang, Dongyan Huang, Xinglong Yue, Yu Zhou, Mingshu Bi., Giant Magnetoimpedance Current Sensor With Spiral Structure Double-Probe. IEEE Trans. Magn. 45 (2009) 1999-2002.

[24] T. Uchiyama, K. Mohri, Y. Honkura, L.V. Panina. Recent Advances of Pico-Tesla Resolution Magneto-Impedance Sensor Based on Amorphous Wire CMOS IC MI Sensor. IEEE Trans. Magn. 48 (2012) 3833-3839.

[25] L.V. Panina, D.P. Makhnovskiy, K. Mohri. Magnetoimpedance in amorphous wires and multifunctional applications: from sensors to tunable artificial microwave materials. J. Magn. Magn. Mater. 272 (2004) 1452-1459.

[26] S. E. Lofland, H. García-Miquel, M. Vázquez, S. M. Bragat. Microwave magnetoabsortion in glasscoated amorphous microwires with radii close to skin depth. J. Apll. Appl. Phys. 92 (2002).

[27] H. Garcia-Miquel, M. Vazquez. Ferromagnetic resonance in Co-rich glass-coated amorphous microwires. Phys. B 299 (2001) 225-229.

[28] H. Garcia-Miquel, M.J. Esbri, J.M. Andres, J.M. Garcia, J.M. Garcia-Beneytez, M. Vazquez. Power absorption and ferromagnetic resonance in Co-rich metallic glasses. IEEE Trans. Magn.37 (2001) 561564

[29] S.M. Bhagat, H. Garcia-Miquel, S.E. Lofland. Ferromagnetic resonance and antiresonance in glasscoated amorphous microwires. J. Magn. Magn. Mater. 249 (2002) 274-277.

[30] H. Garcia-Miquel, J. Carbonell, V. Boria, J. Sanchez-Dehesa. Experimental evidence of left handed transmission through arrays of ferromagnetic microwires. Appl. Phys. Lett. 94 (2009) 054103.

[31] H. Garcia-Miquel, J. Carbonell, J. SanchezDehesa. Left handed material based on amorphous ferromagnetic microwires tunable by dc current. Appl. Phys. Lett. 97 (2010) 094102.

[32] G.V. Kurlyandskaya, D. de Cos, S.O. Volchkov. Magnetosensitive transducers for nondestructive testing operating on the basis of the giant magnetoipedance effect: a review. Russ. J. NonDestructive Testing 45 (2009) 377-398.

[33] L.V. Panina, K. Mohri, K. Bushida, M. Noda. Giant magneto-impedance and magneto-inductive effects in amorphous-alloys. J. Appl. Phys. 76 (1994) 6198-6203. 